\title{
Griffbereite Aufbewahrung einer Salbenportion bei Katheterwechsel
}

Beim Wechsel eines suprapubischen Wechselkatheters, eines Nierenfistelkatheters oder der Versorgung eines Urostomas ist die saubere Aufbewahrung einer Salbenportion, etwa von PVBJod, eine logistische Herausforderung - besonders, wenn keine Hilfsperson bereitsteht. Unsere Autoren verraten Ihnen, wie dies auch ohne Hilfe gelingt.

Vor einem Katheterwechsel sollten möglichst alle Materialien bereitgelegt werden. Eine Portion Salbe aus der Tube lässt sich allerdings in der Regel schlecht ablegen und ohne Salbenverlust wieder aufnehmen. Eine Bereitstellung der Salbe vor dem Wechsel empfiehlt sich auch deswegen, weil die Tube nicht kontaminiert werden darf. Ein kleiner Trick erlaubt es, eine Portion Salbe vor dem Eingriff sauber zu entnehmen und ohne Materialverluste und Handschuhwechsel bis zum Gebrauch bei dem abschließenden Verbandswechsel aufzubewahren: Die benötigte Salbenmenge wird vor der Prozedur auf dem Handrücken einer Hand abgestreift. Hier stört sie nicht während des Katheterwechsels bzw. des Verbandwechsels und kann ohne Zeit- und Materialverluste im weiteren Arbeitsablauf genutzt

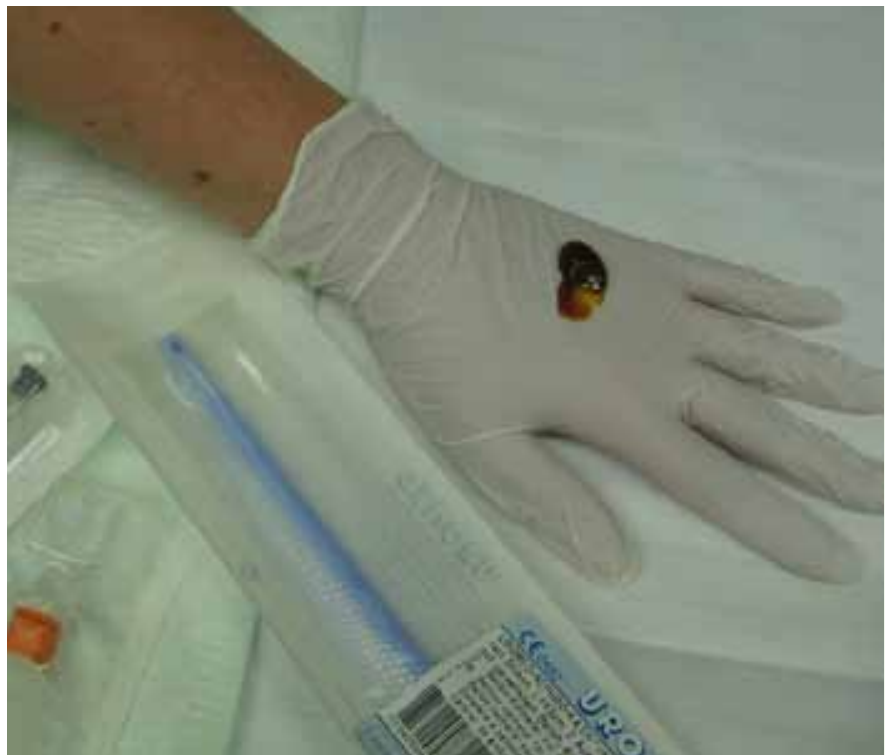

Abb.1 Eine Salbenportion wird vor einem Katheterwechsel sauber entnommen und bis zur Verwendung auf dem Handrücken „geparkt“. werden ( $\bullet$ Abb.1).

PD Dr. Andreas Wiedemann Sr. Sina Richter, Witten

Korrespondenz:

PD Dr. Andreas Wiedemann

Urologische Klinik

Evangelisches Krankenhaus

im Diakoniewerk Ruhr gGmbH

Lehrstuhl für Geriatrie

der Universität Witten/Herdecke

Pferdebachstr. 27

58455 Witten

Tel. 02302/175-2521

Fax. 02302/175-2075

awiedemann@diakonie-ruhr.de 